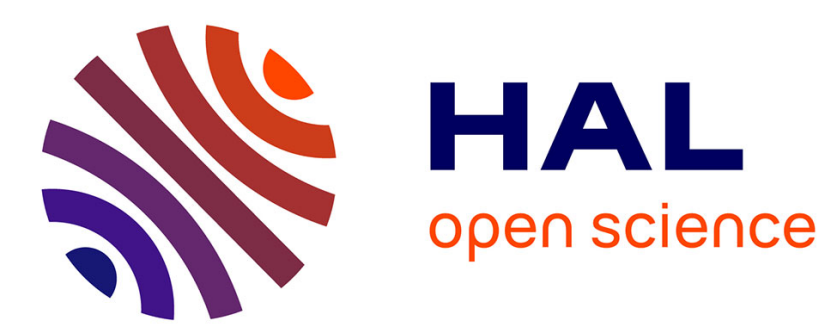

\title{
Les inscriptions du calice et de la patène de l'abbé Pélage au Louvre
}

\author{
Robert Favreau
}

\section{To cite this version:}

Robert Favreau. Les inscriptions du calice et de la patène de l'abbé Pélage au Louvre. Comptesrendus des séances de l'Académie des inscriptions et belles-lettres, 1992, 137 (1), pp.31-48. 10.3406/crai.1993.15179 . halshs-03203107

\section{HAL Id: halshs-03203107 https://shs.hal.science/halshs-03203107}

Submitted on 20 Apr 2021

HAL is a multi-disciplinary open access archive for the deposit and dissemination of scientific research documents, whether they are published or not. The documents may come from teaching and research institutions in France or abroad, or from public or private research centers.
L'archive ouverte pluridisciplinaire HAL, est destinée au dépôt et à la diffusion de documents scientifiques de niveau recherche, publiés ou non, émanant des établissements d'enseignement et de recherche français ou étrangers, des laboratoires publics ou privés. 


\section{Les inscriptions du calice et de la patène de l'abbé Pélage au} Louvre

Robert Favreau

Citer ce document / Cite this document :

Favreau Robert. Les inscriptions du calice et de la patène de l'abbé Pélage au Louvre. In: Comptes rendus des séances de l'Académie des Inscriptions et Belles-Lettres, $137^{\mathrm{e}}$ année, N. 1, 1993. pp. 31-48;

doi : https://doi.org/10.3406/crai.1993.15179

https://www.persee.fr/doc/crai_0065-0536_1993_num_137_1_15179

Fichier pdf généré le 21/05/2018 


\title{
COMMUNICATION
}

\author{
LES INSCRIPTIONS DU CALICE
}

ET DE LA PATĖNE DE L'ABBÉ PÉLAGE AU LOUVRE, PAR M. ROBERT FAVREAL; CORRESPONDANT DE L'ACADEMIE

Le musée du Louvre a acquis en 1886, à la vente de la collection Charles Stein', un calice et une patène en argent doré, qui sont bien connus des historiens et des historiens de l'art pour avoir été présentés dans des expositions en 1878,1880,1936,1970, et avoir fait l'objet de diverses études ou mentions ${ }^{2}$, sans que pour autant on puisse parler à leur sujet d'un travail approfondi. Ils sont exposés au Louvre avec la mention : Meuse ou Espagne ? Vers 1200 (fig. 1). À ce dossier l'examen des deux inscriptions peut apporter une utile contribution à laquelle je voudrais ici m'attacher.

Le calice est en argent doré en partie. Le nœud est découpé à jour, et est orné des symboles des évangélistes au milieu d'entrelacs. L'inscription gravée autour du pied du calice indique que l'œuvre a été commandée par l'abbé Pélage en l'honneur de saint Jacques, PELAGIUS ABBAS ME FECIT AD HONOREM SANCTI JACOBI APOSTOLI (fig. 2a, b). Le nom de Pélage est celui-là même du premier héros de la " reconquête " - " reconquista " - de la péninsule sur les Musulmans qui s'en étaient emparés en 711. Ancien dignitaire de la cour du roi wisigoth de Tolède, Pélage s'était réfugié dans ces montagnes des Asturies et de la Galice, dont les habitants n'avaient jamais accepté une domination étrangère, pas plus des Romains, qui marquèrent fort peu la région, que des Wisigoths eux-mêmes, et qui n'étaient pas

1. Le 10 mai 1886, a la galerie Georges-Petit (Catalogue de la collection Charles Stein mise en vente $10-14$ mai 1886 , p. $149-150, \mathrm{n}^{\circ} 194$ ) pour la somme de $7100 \mathrm{~F}$. Je remercie très vivement $\mathbf{M}^{\text {me }}$ Danielle Gaborit-Chopin, conservateur au département des objets d'art du Louvre, de m'avoir donné accès au dossier concernant ces deux pièces.

2. Ch. Rohault de Fleury, La Messe. Études archéologiques sur ses monuments, IV, Paris, s.d., p. 126 et pl. CCCXIII ; Alfred Darcel, "Le Moyen Âge et la Renaissance au Trocadéro ", Gazette des Beaux-Arts, octobre 1878, p. 541 (reprod., p. 543) ; Charles Davillier, Recherches sur l'orfèvrerie en Espagne au Moyen Age et à la Renaissance, Paris, 1879, p. 36-38, et Les arts décoratifs en Espagne au Moyen Âge et à la Renaissance, Paris, 1879, p. 10-12; Alfred Darcel, * La collection Charles Stein ", Gazette des Beaux-Arts, février 1886, p. 120 ; Émile Molinier, * Le calice de l'abbé Pelage au Musée du Louvre ", Gazette archéologique, 1888, p. 308-311, pl. 39, et Histoire générale des arts appliqués à l'industrie, t. IV, Paris, 1901, p. 195 ; J.-J. Marquet de Vasselot, Musée du Louvre. Orfèvrerie, émaillerie et gemmes du Moyen Âge au XVII' siècle, Paris, 1914, p. 7, ${ }^{\circ} 24$; Joseph Braun, Das christliche Altargerät in seinem Sein und in seiner Entwicklung, pl. 43, n 152 ; Angela Franco Mata, * Arte 1200. Cáliz y patena del abad Pelagio », Diario de León, 19 février 1989. 


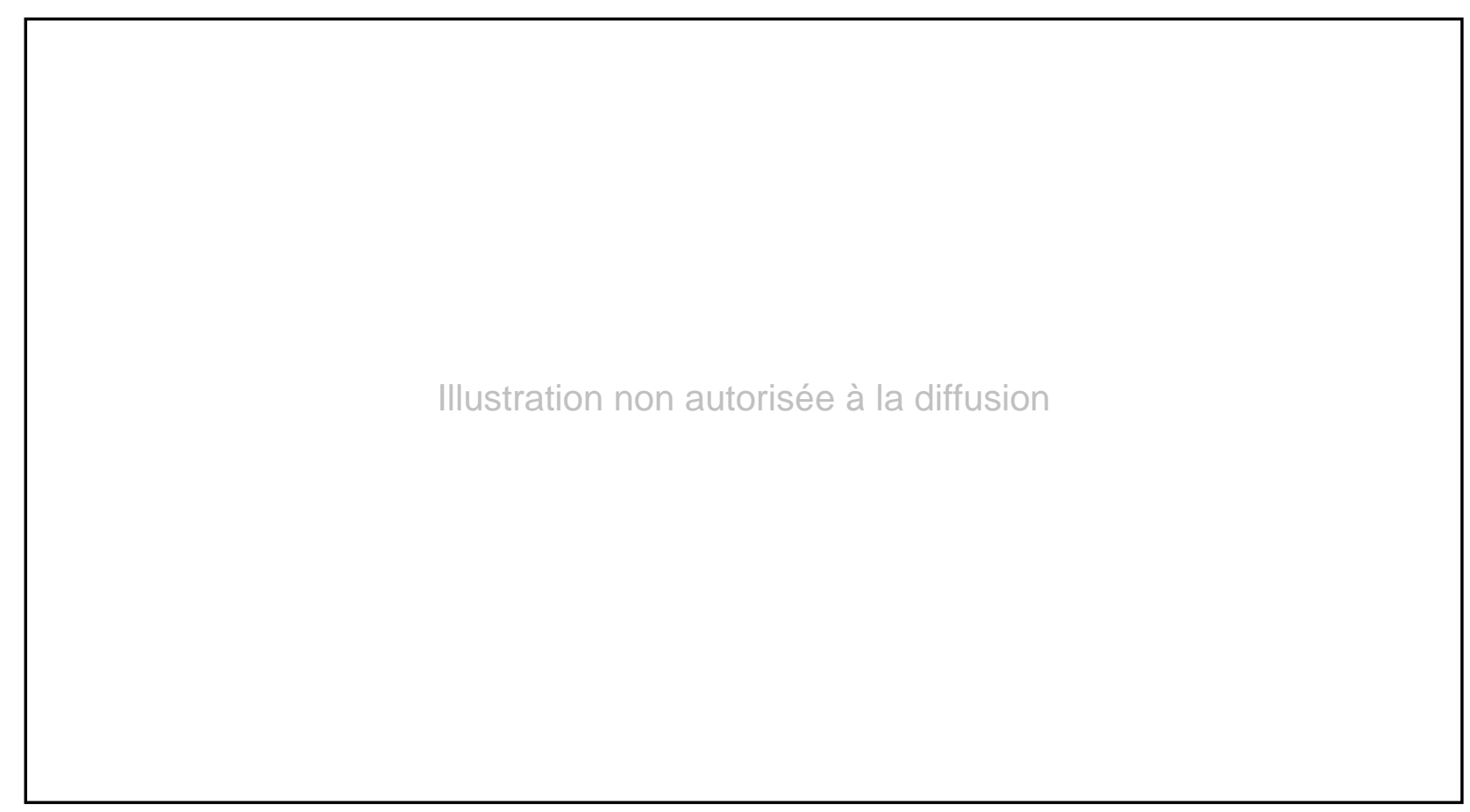

FIG. 1. - Calice et patène de l'abbé Pêlage.

Musée du Louvre, Département des Objets d'art. (C. photo R. M. N.)

prêts à se soumettre aux Musulmans, même si ceux-ci s'étaient installés à Gijon, sur la côte cantabrique. Envoyé un temps en otage à Cordoue, Pélage s'échappa, prit la tête des montagnards du nord-ouest de la péninsule, et battit les Musulmans au printemps de 722 , à Covadonga, à l'est des Asturies. Dès lors les chrétiens retrouvèrent confiance, reprirent Astorga en 749, fondèrent plusieurs monastères, tandis qu'un royaume des Asturies s'organisait, et fixait sa capitale à Oviedo. Au début $d u x^{e}$ siècle l'avancée vers le sud conduisit à transfêrer le centre du royaume à León. Ce fut aussi à cette époque, sous le roi Ordoño II et en cette région que s'implanta le culte de saint Pélage de Cordoue. Donné en otage aux Musulmans, le jeune Pélage avait refusé de céder aux avances du calife et de se rallier à la foi musulmane, ce qui lui avait valu d'être décapité en 925. La région du nord-ouest de la péninsule (fig. 3) où s'était constitué le royaume chrétien d'Asturies-León avait déjà servi de refuge aux moines de Cordoue fuyant la domination musulmane, ce que rappellent deux belles inscriptions de 913 et 921 . La première, aujourd'hui perdue, se trouvait au monastère de San Miguel de Escalada, à 28 kilomètres à l'est de León. Elle nous dit qu' " en ce lieu d'ancienneté dédié en l'honneur de l'archange Michel, construit de simple appareil, bientôt tombé en ruines et longtemps demeuré à l'abandon, l'abbé Alphonse vint de son pays de Cordoue avec ses compagnons. Il releva l'édifice de ses ruines, sous le valeureux et sérénissime prince Alphonse. 
FIg. 2, a et b. - Détails du calice de l'abbé Pélage.

Musée du Louvre, Département des Objets d'art. (C. Photo R. M. N.) 


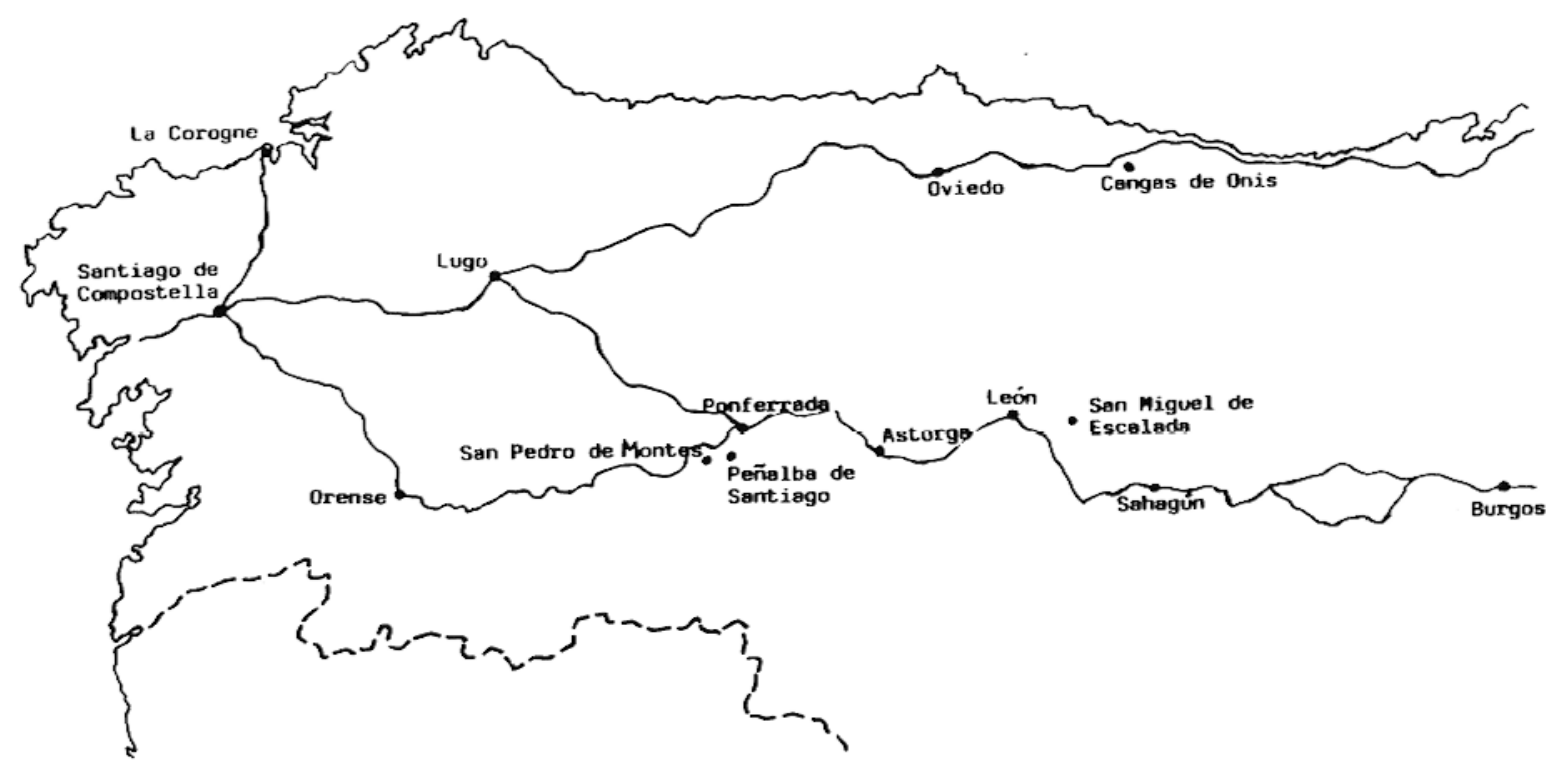

FIG. 3. - Carte du nord-ouest de la péninsule Ibérique.

Le nombre des moines augmentant, ce temple est alors érigé depuis ses fondements, agrandi de toutes parts et admirablement décoré, et ceci, non sur l'ordre du prince ou la pression du peuple, mais par les soins vigilants de l'abbé Alphonse et de ses frères. Ces travaux sont achevés en douze mois au temps du roi Garcia et de la reine Mumadona, en l'an de l'ère 951 (913), et le temple est consacré par l'évêque Gennade le 12 des calendes de décembre "3. La seconde, gravée sur un marbre blanc, conservée à l'extérieur de l'église abbatiale romane de San Martin de Castañeda, à droite de la porte, est très semblable à la précédente : "Ce lieu d'ancienneté dédié en l'honneur de saint Martin, construit de simple appareil, demeura

3. Jaime Federico Rollan Ortiz, * Iglesias mozarabes leonesas «, dans León, $3^{e}$ éd., León, 1987, p. 90 :

ISTE LOCUS ANTIQUITUS MICHAELIS ARCHANGELI HONORE DICATUS BREVI OPERE INSTRUCTUS, POST RUINIS ABOLITUS, DIU MANSIT DIRUTUS, DONEC ADEFONSUS ABBA CUM SOCIIS ADVENIENS A CORDUVENSI PATRIA EDIS RUINAM EREXIT SUB VALENTE SERENISSIMO ADEFONSO PRINCIPE MONACHORUM NUMERO CRESCENTE DEMUM HOC TEMPLUM DECORUM MIRO OPERE A FUNDAMINE EXUNDIQUE AMPLIFICATUM ERIGITUR NON JUSSU IMPERIALI VEL OPPRESSIONE VULGI SED ABBATIS ADEFONSI ET FRATRUM INSTANTE VIGILANTIA DUODENIS MENSIBUS PERACTA SUNT HAEC OPERA, GARSEA SCEPTRA REGNI PERAGENS MUMADOMNA CUM REGINA ERA DCCCCLI. SACRATUMQUE TEMPLUM AB EPISCOPUM IENNADIUM XII KAL DECEMBRIUM.

Étant donné qu'une dédicace a lieu ordinairement un dimanche, on a retenu à côté du samedi 20 novembre 913 , la date du 20 novembre 914 . L'inscription est rapportée par lc frc̀rc Manucl Risco dans l'España sagrada, XXXV, p. 310-311. Voir aussi E. Huebner, Inscriptionum Hispaniae christianae supplementum, Berlin, 1900, n 469, p. 107 ; Jacques Fontaine, L'art préroman-hispanique ${ }^{\star}$. L'art mozarabe, La Pierre-qui-Vire, 1977, p. 83 ; Vicente Garcia Lobo, Las inscripciones de San Miguel de Escalada. Estudio critico, Barcelone, $1982, n^{\circ} 8$, p. 64-65. 
longtemps en ruines jusqu'à ce que l'abbé Jean vienne de Cordoue. Celui-ci redonna au temple son caractère sacré, releva la ruine de l'édifice depuis ses fondements, arrachant la pierre à la terre et la mettant en ouvre. Ce fut non sur l'ordre du prince mais sur les instances vigilantes de ses frères que ces travaux furent achevés en cinq mois, sous le règne d'Ordoño, en l'ère 9594. "Dès 951 on connaît des reliques de saint Pélage à San Martin de Salas dans les Asturies's . Le corps du saint fut transféré à León en 967 , puis, lorsque la menace musulmane d'Al Manzor fut trop pressante, à Oviedo en 985, où il est encore aujourd'hui vénéré.

Bien que les mérites du saint aient été tôt connus en Occident puisque dès la fin du $x^{e}$ siècle la moniale Hrotsvitha, à Gandersheim en Saxe, écrit sa Passio en vers 6 , le nom de Pélage se rencontre presque exclusivement dans la péninsule Ibérique. Dans son répertoire des noms de personne sur le territoire de l'ancienne Gaule, Marie-Thérèse Morlet cite une fois le prénom de Pélage, en 1088, dans le cartulaire de SaintVictor de Marseille, mais il s'agit d'une charte d'Alphonse VI, roi de Galice, des Asturies, de León'? ${ }^{7}$ On n'en trouve aucun exemple dans les tables onomastiques des neuf volumes du Recueil des historiens des Gaules et de la France qui intéressent les $\mathrm{X}^{\mathrm{e}}$-XII ${ }^{\mathrm{e}}$ siècles. Bien plus, dans la péninsule Ibérique, il semble fortement localisé en sa partie nord-ouest. Ainsi un sondage le montre absent dans les cartulaires de Navarre, Aragon, Catalogne, alors que, dans tel cartulaire d'Oviedo, on en rencontre des dizaines d'exemples, et si on prend

4. Maximino Gutierrez Alvarez, Las inscripciones medievales de la provincia de Zamora, Université de León, thèse de doctorat, II, 1991, n² 2, p. 346-347 (dactyl.) :

[HIC LOCUS ANTIQUITUS MARTINUS SANCTUS HONORE

DICATUS, BREVI OPERE INSTRUCTUS, DIU MANSIT BIRUTUS,

DONEC JOHANNES ABBA A CORDOBA VENIT ET HIC TEMPLUM LITAVIT

EDIS RUGINAM A FUNDAMINE EREXIT ET ACTE SAXE EXARABIT

NON IMPERIALIBUS JUSSUS ET FRATRUM VIGILANTIA INSTANTIBUS

DUO ET TRIBUS MENSIBUS PERACTI SUNT HEC OPERIBUS

HORDONIUS PERAGENS SCEPTRA ERA NOBI ET SEMIS CENTENA NONA.

Voir aussi Antonio Vinayo Gonzalez, L'ancien royaume de León roman. La Pierre-qui-Vire, 1972 , p. 334.

San Martin de Castañeda est dans la Sierra de la Cabrera, au nord-ouest de Puebla de Sambria.

5. Ciriaco Miguel Vigil, Asturias monumental epigráfica y diplomática datos para la historia de la provincia, Oviedo, 1887, p. $58, \mathrm{n}^{\circ} \mathrm{Eb} 13$, pl. Eb VI.

6. AASS, juin, VII (Paris et Rome, 1867), p. 181-200 ; P.L., 137, col. 1093-1102. On verra aussi Manuel C. Diaz y Diaz, "La pasion de San Pelago y su difusion ", Anuario de estudios medievales, 6, 1969, p. 97-112.

7. Marie-Thêrèse Morlet, Les noms de personne sur le territoire de l'ancienne Gaule du $V^{e}$ au XII siècle. II. Les noms latins ou transmis par le latin, Paris, 1972, p. 89.

8. Pas de Pélage aux tables pour la Coleccion diplomatica de Obarra (siglos XI-XII), par Angel J. Martin Duque, Saragosse, 1965 (Navarre), El " Llibre Blanch " de Santas Creus (Cartulario des siglo XII) par Federico Udina Martorell, Barcelone, 1947 (Catalogne), la Documentacio Pinatense del siglo XII, par Maria Ascension Lluch Adelantado, et la 
les listes épiscopales de toute la péninsule, on ne trouve d'évêques du nom de Pêlage, entre la fin du $x^{e}$ et la fin du XIr ${ }^{e}$ siècle, qu'à Oviedo, León, Astorga, Compostelle, Mondoñedo et Lugo, Braga, Porto, Coïmbra et Evora. Tous ces évêchés entretiennent d'êtroites relations. L'élégante inscription de dédicace de la petite église asturienne de Val de Dios en 893 mentionne la présence des évêques de Coïmbra, Compostelle, Mondoñedo, Lugo, Astorga, ainsi que de Lamego et de Saragosse, ce dernier réfugié à Oviedo9 : celle de San Pedro de Montes, en León, en 919, cite les évêques d'Astorga, de Mondoñedo, de León et de Salamanque ${ }^{10}$, celle de Castiello en Raices dans les Asturies, les noms de trois évêques présents à Val de Dios en 893, ceux de Compostelle, Coïmbra et Lugo"1.

Les listes abbatiales sont bien trop fragmentaires pour qu'on puisse déterminer l'abbaye d'où proviendraient le calice et la patène. Il faut donc étudier la seconde partie de l'inscription du calice qui le dit fait " en l'honneur de saint Jacques apôtre ". On vénère dès 627 des reliques de saint Jacques en Espagne, au témoignage d'une inscription trouvée en 1947 à Merida12. Une hymne liturgique fut composée en l'honneur du saint à la fin du virre siècle. Avant 829 on découvrit son tombeau, près d'Iria, une ville épiscopale de Galice, au * champ de l'étoile ", Campus stellae. Très peu de temps après Florus de Lyon écrit dans son Martyrologe : "Les ossements sacrés de ce très bienheureux apôtre, transférés en Espagne, y sont vénérés par ces peuples avec une extraordinaire dévotion, à l'extrêmité du pays. "Le culte de l'apôtre aura désormais une grande importance dans le petit royaume chrétien des Asturies, tout tendu vers la reconquête ${ }^{13}$, royaume dont la capitale se fixa à Oviedo $\mathrm{au}$ début $\mathrm{du} \mathrm{Ix}^{\mathrm{e}}$ siècle, et fut transférée à León au début du $\mathrm{x}^{\mathrm{e}}$ siècle. Des reliques du saint sont mentionnées dans diverses églises des Asturies et León, avec ou sans la mention de la qualité apostolique ${ }^{14}$.

Documentacio particular Pinatense de 1063 a 1095, par Marfa Pilar Jerez Martin, travaux de l'Université de Valence, 1959-1960 (San Juan de la Peña, Aragon). On trouve un Pelaio Perez en 1172 et 1173 dans le Cartulario del Monasterio de Santa Maria de Huerta, par José Antonio Garcia Lujan, Monasterio de Santa Maria de Huerta, 1981 (province de Soria). Pour Oviedo, j'ai parcouru la Coleccion diplomatica del monasterio de San Vicente de Oviedo de Pedro Floriano Llorente, Oviedo, 1968 (pas d'index des noms).

9. Vigil, op. cit., p. 595-596. $\mathrm{n}^{\circ} \mathrm{Ub} 10$, pl. Ub V.

10. Emile Huebner, Inscriptiones Hispaniae christianae, Berlin, 1871, p. 79, n 245.

11. Vigil, op. cit., p. $336, \mathrm{n}^{\circ} \mathrm{N} 1$.

12. José Vives, Inscriptiones cristianas de la España romana y visigoda, Barcelone, 1969, p. 314-315, n० 548 ; Santiago de Compostela. 1000 ans de pèlerinage européen, Gand, 1985, p. $202, n^{\circ} 2$.

13. J. Fontaine, L'art préroman hispanique ${ }^{\star}$, La Pierre-qui-Vire, 1973, p. 253.

14. D'après les inscriptions à Santa Eulalia de Doriga, et Santiago de Civea, à San Miguel de Escalada (avec le mot * apôtre »), à San Pedro de Montes, à San Miguel de Quiloño ou à la cathédrale d'Oviedo (sans le mot apôtre). 
Mais si on s'attache à l'expression " en l'honneur de saint Jacques apôtre " on ne trouve, dans les inscriptions de la région, que quatre exemples. Le premier est celui qui a fourni sans doute un modèle, puisqu'il s'agit du texte gravé sur la croix d'or, imitée de la croix des anges, que le roi des Asturies Alphonse III offrit, de concert avec sa femme, la reine Chimène, à la cathédrale de Saint-Jacques de Compostelle en 874 :

+OB HONOREM SANCTI JACOBI APOSTOLI OFFERUNT
FAMULI DEI ADEFONSUS PRINCEPS CUM CONJUGE
SCEMENA REGINA.
HOC SIGNO TUETUR PIUS HOC SIGNO VINCITUR INIMICUS.
HOC OPUS PERFECTUM EST IN ERA DCCCC ${ }^{\text {DUODECIMA }}{ }^{15}$.

On retrouve ici associés le culte de l'apôtre Jacques et la célèbre " croix d'Oviedo " qui fut le symbole primitif de la "reconquista ".

Le deuxième exemple est donné par l'inscription de la croix que le roi Ramire II offrit, sans doute vers 940 , à l'abbaye de Santiago de Peñalba, et qui se trouve aujourd'hui au Museo Arqueológico de León : IN NOMINE DOMINI NOSTRI JHESU CHRISTI, OB ONOREM+SANCT[I] JACOBI APOSTOLI, RANEMIRUS REX OF[E]RT ${ }^{16}$.

Avec Peñalba on est dans le diocèse d'Astorga, à une vingtaine de kilomètres au sud de Ponferrada où passera le grand chemin de SaintJacques, dans cette partie des montagnes de León qu'avait illustrée saint Gennade. Moine de Sahagun, Gennade s'était retiré dans ces montagnes où, autour de 900 , il avait restauré un ancien oratoire, San Pedro de Montes, comme nous l'explique une inscription à la fois commémorative et de dédicace : " $D$ 'insignes mérites saint Fructueux, après avoir fondé l'abbaye de Compludo, fit en ce lieu un modeste oratoire placé sous le patronage de saint Pierre. Après lui saint Valérien, qui ne lui cédait en rien pour les mérites, agrandit l'église. En dernier lieu le prêtre Gennade, avec douze frères, le restaura en l'an de l'ère 933 (895). Devenu évêque, il l'érigea finalement, depuis les fondations, de façon magnifique, poussé non par la pression populaire mais par l'assurance de la récompense et avec l'aide des frères de ce monastère. Ce temple fut consacré par quatre évêques, Gennade d'Astorga, Sabarius de Mondoñedo, Fruminius de León et Dulcide de Salamanque, en l'an de l'ère 944 , le neuf des calendes de novembre " (24 octobre 906$)^{17}$.

15. Huebner, Inscriptiones Hispaniae christianae, p. 81, $\mathrm{n}^{\circ} 252$.

16. Manuel Gomez-Moreno, Catalogo monumental de España. Provincia de León (1906-1908), Madrid, 1925, p. 145 ; J. Fontaine, L'art préroman hispanique ${ }^{\star \star}$. L'art mozarabe, p. $372 \mathrm{et}$ fig. 44-45. Ramire II a régné de 930 à 950 ; on connaît de lui des dons faits à Peñalba en 940 .

17. Huebner, Inscriptiones Hispaniae Christianae, p. $79, \mathrm{n}^{\circ} 245$ :

INSIGNE MERITIS, BEATUS FRUCTUOSUS, POSTQUAM COMPLUTENSE CONDIDIT COENOBIUM, SUB NOMINE S. PETRI BREVI OPERE IN HOC LOCO FECIT ORATORIUM; POST QUEM NON IMPAR MERITIS VALERIUS SANCTUS OPUS ECCLESIAE DILATAVIT. NOVISSIME GENNADIUS PRESBYTER CUM DUODECIM FRATRIBUS RESTAURAVIT ERA DCCCCXXXIII; PONTIFEX 
Il établit à Montes une autre chapelle en l'honneur de saint André, et, comme il le dit dans son testament, " un peu à l'écart il fonda, en mémoire de saint Jacques, un troisième monastère qui s'appelle Peñalba ", Pinna Alba, la Roche Blanche, en raison des blocs calcaires qui le surplombent ${ }^{18}$. Ce fut là qu'il mourut, après avoir déposé la charge d'évêque d'Astorga qui lui avait été confiée. Il léguait à ses fondations calices, patènes, croix, et livres liturgiques, et sa bibliothèque qui devait être commune entre elles ${ }^{19}$. Son successeur Salomon acheva l'établissement de l'abbaye de Santiago de Peñalba en 937. Le troisième exemple de l'expression * en l'honneur de saint Jacques apôtre » est à nouveau à Santiago de Peñalba lors de la consécration de $1105^{20}$, qui est sans doute le fait de l'évêque d'Astorga, Pélage. Le quatrième exemple figure aussi dans une inscription de dédicace, mais dans un contexte plus large et avec une formulation légèrement différente : en 1108, l'évêque d'Oviedo consacre trois autels en l'église San Adriano de Tuñon (Asturies), le premier IN HONORE BEATORUM MARTIRUM ADRIANI ET NATALIE, celui de droite IN HONORE BEATORUM PETRI ET PAULI APOSTOLORUM, celui de gauche IN HONORE BEATI JACOBI APOSTOLI'1. Finalement il y a trois expressions seulement parfaitement comparables à celle du calice de l'abbé Pélage, celle de Saint-Jacques de Compostelle et les deux de Santiago de Peñalba. Les historiens qui ont proposé une localisation pour ce calice et cette patène de l'abbé Pélage ont tous retenu Peñalba. C'est au moins celle qui est la plus probable.

On peut se demander si le commanditaire ou l'artiste a inscrit son nom sur le calice qu'il offrait ou réalisait à une époque particulière et non à une autre. Pour ce qui concerne les calices on trouve les noms gravés sur le calice dès la fin du viII et on les rencontre de façon régulière jusqu'au $\mathrm{xv}^{\mathrm{e}}$ siècle ${ }^{22}$. Il n'y a pas là un élément de

EFFECTUS A FUNDAMENTIS MERIFICE, UT CERNITUR, DENUO EREXIT, NON OPPRESSIONE VULGI SED LARGITATE PRETII ET SUDORE FRATRUM HUJUS MONASTERII. CONSECRATUM EST HOC TEMPLUM AB EPISCOPIS QUATUOR, GENNADIO ASTORICENSE, SABARIO DUMIENSE, FRUMINIO LEGIONENSI ET DULCIDIO SALMATICENSI, SUB ERA NOVIES CENTENA DECIES QUATERNA ET QUATERNA, NONO KALENDARUM NOVEMBRIS.

18. J. Fontaine, op. cit., p. $138-143$ et 416 ; voir aussi les travaux d'Auguste Quintana Prieto, Peñalba (León, 1953), Monografia histórica del Bierzo (Madrid, 1956), Peñalba. Estudio histórico sobre el monasterio berciano de Santiago de Peñalba (León, 1963).

19. Acta sanctorum, mai, V, p. 95.

20. M. Gomez-Moreno, Catalogo monumental de España. Provincia de León, p. 121 : IN ERA CAXIIII POST MILLESIMA ET VII IDUS MARCII CONSECRATA EST HEC ECCLESIA IN HONOREM SANCTI JACOBI APOSTOLI ET PLURIMORUM.

21. Vigil, Asturias monumental..., p. 527-528, n Jb 1, pl. Jb 1.

22. Voir en particulier Victor Elbern, "Der eucharistische Kelch im frühen Mittelalter *, Zeitschrift des deutschen Vereins für Kunstwissenschaft, XVII, 1963, p. 1-76 et 117-188, et Piotr Skubiszewski, * Die Bildprogramme der romanischen Kelche und Patenen ", dans Mettalkunst von der Spätantike bis zum ausgehenden Mittelalter, Berlin, 1982, p. 198-267 (Schriften der Frühchristlich-byzantinischen Sammlung, I) ; Joseph Braun, Das christliche Altargerät in seinem Sein und in seiner Entwicklung, Munich, 1932, p. 170-172. 


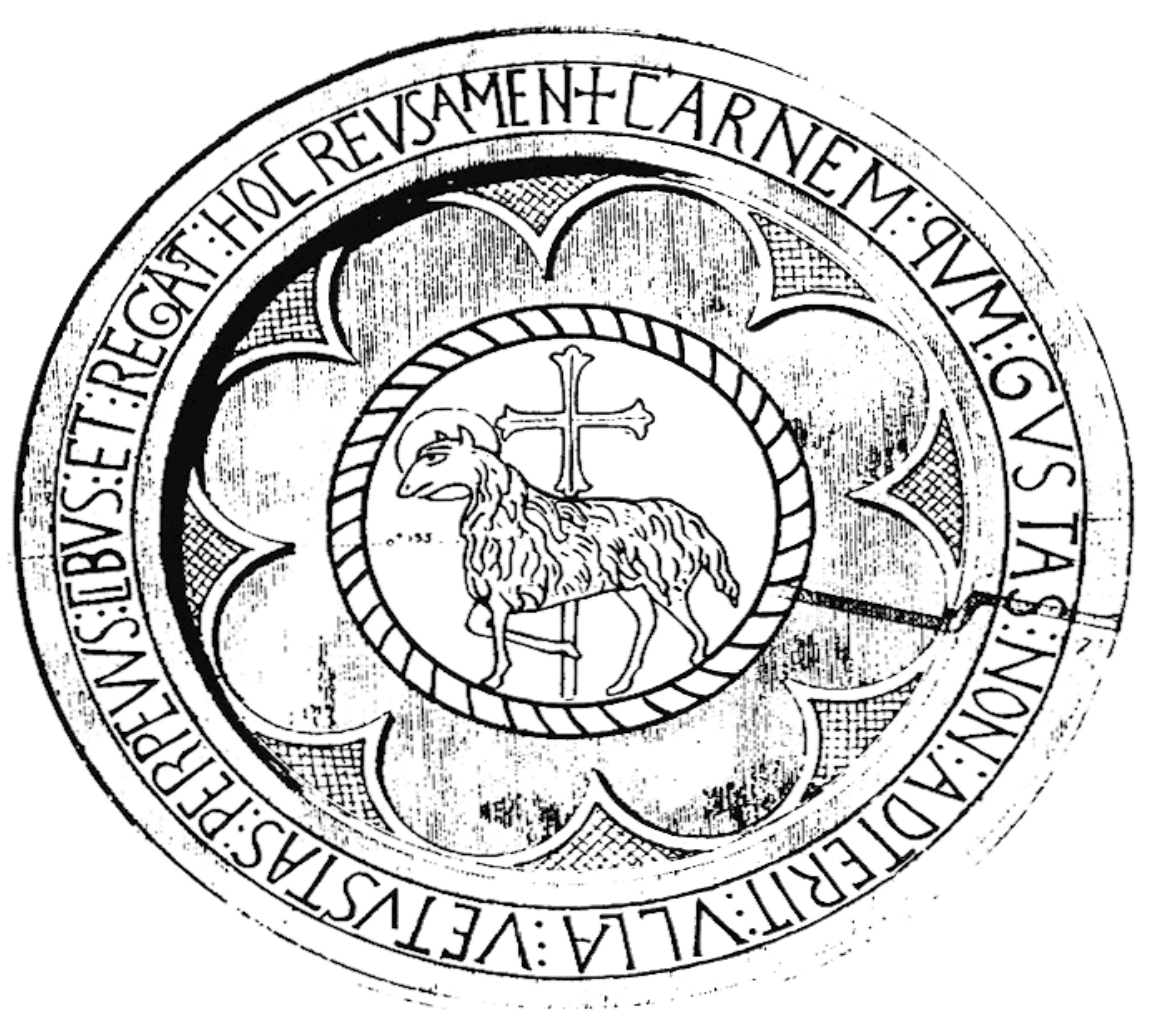

Fig. 4. - La patène de l'abbé Pélage et son inscription : + CARNEM:QUM:GUSTAS:NON:ADTERIT:ULLA:VETUSTAS: PERPETUUS:CIBUS:ET:REGAT:HOC:REUS:AMEN

(Ch. Rohault de Fleury, La Messe. Études archéologiques sur ses monuments, IV, pl. CCCXIII, juin 1882).

datation ni d'ailleurs de localisation, tant la pratique est générale. On voit bien que le commanditaire pensait tirer le plus haut profit en plaçant son nom sur le calice même qui allait servir à la célébration eucharistique.

La patène présente au centre l'agneau pascal nimbé, devant une croix à hampe (fig. 4). Après des lobes rayonnants le bord plat porte une inscription qui n'a guère été étudiée, car elle est restée énigmatique en sa dernière partie. Elle se lit ainsi :

\section{+ CARNEM QUM GUSTAS NON ADTERIT ULLA VETUSTAS PERPETUUS CIBUS ET REGAT HOC REUS AMEN.}

Émile Molinier avait rapporté, sans conviction, en 1888 , la proposition de correction de Julien Havet : "perpetuus cibus en peccatique hoc revocamen "23, et il écrivait en 1901 : "l'inscription présente quelques difficultés d'interprétation, mais cette interprétation n'a pas

23. E. Molinier, * Le calice de l'abbé Pélage au musée du Louvre ", p. 310. 
d'importance pour l'âge du monument ${ }^{24}$. Voilà bien une attitude qui a eu longtemps cours, selon laquelle l'inscription avait comme intérêt principal de fournir, par l'écriture, un argument supplémentaire pour la datation de l'objet ou du monument. Bien plutôt faut-il s'attacher au message de fond que l'inscription nous livre.

La première partie de l'inscription forme un hexamètre léonin riche, dans lequel il faut apporter une légère correction de qum en quam: "La chair que tu manges nulle vieillesse ne l'use ", ou, de façon moins littérale : " sur la chair que tu manges l'âge n'a pas de prise *. Peut-être y a-t-il une allusion au livre de Josué, IX, 12 : « Voici notre pain : il était chaud quand nous en avons fait provision dans nos maisons le jour où nous sommes partis pour venir vers vous ; maintenant le voilà sec et réduit en miette ", vetustate nimia comminuti. Cette fois il ne s'agit plus d'un pain périssable, mais d'une " nourriture perpétuelle ", comme l'affirme le début du second vers, perpetuus cibus. Nous sommes renvoyés à l'évangile de Jean (VI, 56) : "Caro enim mea vere est cibus ", ce que reprendra Paschase Radbert, dans son traité " sur le corps et le sang du Seigneur " : "Caro et sanguis cibus noster efficitur aeternus „25. L'expression figure aussi dans le vitrail des allégories de saint Paul à Saint-Denis commandé par 1'abbé Suger : PERPETUUSQUE CIBUS NOSTER ET ANGELICUS ${ }^{26}$. Pour avoir un hémistiche exact il faut, dans la patène de l'abbé Pélage, corriger le et en est : "Perpetuus cibus est ", ce qui nous conduit, si le second vers est léonin riche comme le premier, à attendre une finale en -us est. Même si ces corrections sont évidentes et peu importantes, elles n'en traduisent pas moins la maladresse de l'auteur de l'inscription, maladresse qui devient criante lorsque l'on considère la fin de l'inscription, qui n'a pas la finale attendue, et surtout qui n'a aucun sens.

Dans l'énigmatique dernière partie du vers, le reus peut faire penser à l'avertissement de Paul aux chrétiens de Corinthe : " qui mange ce pain et boit à la coupe du Seigneur indignement se rendra coupable (reus) envers le Corps et le Sang du Seigneur " (1 Cor. XI, 27), texte qui a d'ailleurs été gravé sur la croix de Liessies vers 1160-117027. Mais le sens de l'inscription n'est pas là, et il doit être cherché dans le dossier de la controverse bérengarienne au sujet de ce qu'on appellera, à cette époque même, la * transsubstantiation ", c'est-à-dire le changement (creari) réel (res) du pain en la chair du

24. Id., Histoire générale des arts appliqués à l'industrie, IV, p. 195, n. 3.

25. P.L., 120, c. 1311 .

26. Louis Grodecki, Les vitraux de Saint-Denis. Etude sur le vitrail au XII' siècle, Paris, 1976, p. 93 et 144 (Corpus vitrearum medii aevi. France. Série * Études *. Volume I).

27. L'art roman. Exposition organisée par le gouvernement espagnol sous les auspices du Conseil de l'Europe. Catalogue, Barcelone, 1961, p. 261-262, n०413. 
Christ, bien que demeurent les apparences (species) du pain, pour employer un vocabulaire qui est celui-là même des auteurs qui ont combattu l'enseignement de Bérenger ${ }^{28}$. Un écrit anonyme de Melk, rédigé peu après 1135 , le $D e$ scriptoribus ecclesiasticis, nous rapporte que Bérenger dut renoncer à son " hérésie " au cours d'un concile réuni à Rome par le pape Nicolas, et qu'il aurait confirmé sa foi catholique dans le sacrement du corps et du sang du Seigneur Sauveur par ces vers :

Constat in altari carnem de pane creari;

Ipsa caro Deus est; qui negat hoc reus est ${ }^{29}$,

" il est certain que sur l'autel à partir du pain est créée la chair, cette chair même est Dieu ; qui le nie est coupable ». Il est évident que sur la patène de l'abbé Pélage le second vers devrait se terminer par cette mise en garde, qui negat hoc reus est, qui traduit, dans ce contexte, une affirmation forte d'orthodoxie sur la transsubstantiation. Le regat hoc reus est, amen n'a pas de sens, et il lui manque un pied pour former un hexamètre. Il devait d'ailleurs s'agir ici d'un distique élégiaque léonin riche, comme dans le distique attribué à Bérenger.

Le distique qu'aurait prononcé Bérenger ne se trouve pas dans la rétractation de l'écolâtre de Tours à Rome en 1059, ni dans les autres rétractations connues ${ }^{30}$, mais cette formulation a eu une fortune certaine. En effet, on le trouve presque tel quel sur une patène à Götene en Suède :

CONSTAT IN ALTARI CARNEM DE PANE CREARI

ISTE CIBUS DEUS EST QUI NEGAT HOC REUS EST ${ }^{31}$,

et le premier vers a êté gravé vers 1200 sur la patène de la cathédrale de Fritzlar :

CONSTAT IN ALTARI CARNEM DE PANE CREARI.

DA, DEUS, IN REBUS QUOD SUMITUR IN SPECIEBUS ${ }^{32}$.

28. Anselme de Cantorbery dans sa lettre107, De corpore et sanguine Domini : * nec remanere substantiam panis et vini speciem tamen intellegendum est remanere * (P.L., 159, c. 255-256); Rupert de Deutz, De Trinitate ; " specie panis * (P.L., 167, c. 1663); Gerhohus, prévôt de Reichersberg (Autriche), Liber de gloria et honore filii hominis : « non solum sacramento tenus, ut voluit quidam Berengarius..., sed in rei veritate, ita ut ipsius Christi verum corpus de virgine sumptum in altari praesentetur... * (P.L., 194, c. 1117).

29. P.L., 213, c. 979.

30. Sacrosancta concilia, éd. Labbé et Cossart, IX, 1671, c. 1011-1012; Robert Somerville, « The Case Against Berengar of Tours. A New Text ", dans Studi Gregoriani, IX, 1972, p. 68-69.

31. Joseph Braun, Das christliche Altargerät..., p. 229.

32. Die Inschriften der Stadt Fritzlar, ed. Theodor Niederquell, Munich, 1974, p. 9-10, $n^{\circ} 8$ (Die deutschen Inschriften, 14. Heidelberg Reihe 5 band). 
Le distique se rencontre, avec quelques variantes, dans de nombreux manuscrits contenant des pièces sur l'eucharistie : Constat in altari carnem de pane creari dans une série de manuscrits de la Bibliothèque nationale, de la Bibliothèque Mazarine à Paris, de la Bibliothèque de Munich ${ }^{33}$, constat in altari carnem de pane sacrari dans des manuscrits de Bâle, Oxford, Vienne ${ }^{34}$, avec illa caro Deus est, qui dubitat reus est, ou iste cibus Deus est, qui negat hoc reus est pour le second vers, moins fréquemment représenté. Le distique attribué à Bérenger se pose en une simple affirmation de foi, et les historiens de la liturgie ont souligné qu'à partir de la réaction anti-bérengarienne, un des thèmes importants de la théologie eucharistique fut qu'il fallait croire sans chercher à comprendre ${ }^{35}$.

Ce fut un poème eucharistique de cette sorte qui a été la source de l'inscription du calice de l'abbé Pélage. Un manuscrit de la bibliothèque de l'Université de Copenhague, venant de l'abbaye bénédictine SS. Côme-et-Damien de Liesborn en Westphalie, et composé vers la fin du $\mathrm{XII}^{e}$ siècle, contient une collection de poèmes des $\mathrm{XI}^{\mathrm{e}}$ et $\mathrm{XII}^{\mathrm{e}}$ siècles ${ }^{36}$. On $\mathrm{y}$ trouve au folio $43 \mathrm{v}^{\circ}$ un poème de quatre vers : De corpore Christi :

«Carnem quam gustas non atterit ulla venustas

Perpetuus cibus est, qui negat hoc reus est.

Hec caro, quam comedis, te salvat, si bene credis,

Ut male si credis, indicium comedis. "37

L'abbé Pélage a donc copié les deux premiers vers de ce poème, mais les erreurs, qum pour quam, et pour est, et surtout le regat au lieu de negat montrent que lui-même ou le graveur a bien mal compris le texte. Le manuscrit qu'il a eu sous les yeux n'était sûrement pas

33. Paris, Bibl. nat., ms. latin 3479 , fol. 2 ; ms. latin 3627 , fol. $89 \mathrm{v}^{\circ}$; ms. latin 3630 , fol. 44, $72 \mathrm{v}^{\circ}$; ms. latin 13468, fol. 129 ; Bibl. Mazarine, ms. 1090 (993), fol. 50 ; B. Haureau, « Notice sur le manuscrit 13468 de la Bibliotheque nationale ", dans Notices et extraits des manuscrits de la Bibliothèque nationale, t. 33, 1890, p. 219 ; Théod. von Karajan, * Buch des Rügen *, dans Zeitschrift für deutsches Alterthum, II, 1842, p. 7.

34. Hans Walther, Carmina Medii aevi posterioris latina, II/1 Proverbia sententiaeque latinitatis Medii aevi..., 1 : A-E, Göttingen, 1963, p. 376, n 3219 ; "Florilegium Gottingense ", éd. Ernst Voigt, dans Romanische Forschungen. Organ für romanische Sprachen und Mittellatein, hgg. Karl Vollmoeller, III, 1887, p. 310. On trouve encore Hic panis Deus est, qui negat hunc reus est dans une série de poèmes eucharistiques conservée dans un manuscrit d'Assise du début du xıve siècle (Dom André Wilmart, Auteurs spirituels et textes dévots du Moyen Âge latin. Études d'histoire littéraire, Paris, 1932, p. 414).

35. Pierre-Marie Gy, La liturgie dans l'histoire, Paris, 1990, p. 190.

36. Paul Lehmann, * Eine Sammlung mittellateinischer Gedichte aus dem Ende des 12. Jahrhunderts *, dans Historische Vierteljahrschrift. Zeitschrift für Geschichtswissenschaft und für lateinische Philologie des Mittelalters, XXX, 1935, p. 20-58 : Copenhague, Univ. Bibl. Ms. Fabr. 81 in- $\mathbf{B}^{\circ}$.

37. Ibid., p. 30 . Il faut évidemment lire dans le quatrième vers judicium (cf. Paul, 1 Cor XI, 29) et non indicium. 
celui qui aujourd'hui est conservé à Copenhague, d'autant plus que ce dernier donne, à tort, venustas au lieu de vetustas qui est la forme, correcte, figurant sur la patène du Louvre. Mais on sait que ces petits poèmes sur l'eucharistie ont été copiés à de nombreuses reprises, et peut-être retrouvera-t-on dans le nord-ouest de la péninsule Ibérique le manuscrit qui a été à la source de l'inscription, manuscrit qui pourrait, d'ailleurs, avoir été apporté de France. En effet, c'est sans doute sous l'influence française que le vers léonin riche trouve une large fortune en Espagne à partir du XII siècle. D'un premier examen, qui ne pourrait être satisfaisant qu'à partir d'un corpus général, il ressort que le vers léonin riche apparaît dans les inscriptions de Galice dans un autel portatif de l'abbaye de Celanova au temps de l'abbé 'Pierre (1090-1118) [Orense, Musée diocésain], dans celles des Asturies en 1129 à Oviedo, en 1138 à Cornellana, dans celles du royaume de León à la fin du XII ${ }^{e}$ siècle à Sahagun, en 1169 à San Miguel de Escalada, à Astorga en 1141 et surtout en 1132 à Santiago de Peñalba, dans l'épitaphe de l'abbé Étienne, d'origine française, épitaphe composée de dix distiques élégiaques léonins riches ${ }^{38}$. Or on a estimé que c'est au début du XII' siècle que l'abbaye de Peñalba a été rattachée à l'ordre de Cluny 39 . En Castille, de même, le léonin riche n'apparaît qu'en 1175 à Villemartín. La formule sera dès lors très prisée et se poursuivra au moins jusqu'au milieu du XIV siècle dans les Asturies. La métrique apporte donc ici un nouvel élément de datation, en même temps qu'elle traduit une influence très probablement française.

Il reste à examiner l'écriture de ces deux inscriptions. Une lettre est caractéristique, le $\mathrm{T}$ wisigothique de REGAT dans l'inscription de la patène. Cette forme se trouve dans une inscription de Saint-Genisdes-Fontaines en 1019-102040, mais elle ne dépasse qu'à peine les Pyrénées, au point qu'on peut la dire propre à la péninsule Ibérique. Dans les archives de la cathédrale de León, dans celles de Sahagun, les documents en écriture wisigothique s'observent jusqu'en 1128 ; pour le monastère de Lorenzana (Coruña), cette écriture est encore

38. España sagrada..., XVI. De la santa iglesia de Astorga, éd. Henrique Florez, Madrid, 1787, p. 41 . La seule exception trouvée à cet examen métrique est l'épitaphe du roi Ordoño, mort en 894, à la cathédrale de León. Cette épitaphe comporte deux distiques élégiaques léonins riches, séparés par deux hexamètres léonins riches (E. Huebner, Inscriptiones Hispaniae christianae, $\mathrm{I}$, p. $78, \mathrm{n}^{\circ} 242$ ). Je crois que cette épitaphe ne peut être du $\mathrm{IX}^{\mathrm{e}}$ siècle mais a dû être réalisée au $\mathrm{XII}^{\mathrm{e}}$ ou au Xille siècle.

39. Antonio Linage Conde, Los origines del monacato benedictino en la peninsula iberica. II. La difusion de la « regula Benedicti *. León, 1973, p. 713-717 (Coleccion Fuentes y estudios de la historia Leonesa, 10).

40. Corpus des inscriptions de la France médiévale. 11. Pyrénées-Orientales, par R. Favreau, J. Michaud, B. Mora, Paris, 1986, p. 138. 
utilisée en 1124 ; pour San Salvador de Oña (Burgos), on la trouve de la fin du $x^{e}$ siècle à $1072^{41}$. Bernhard Bischoff note la disparition de la nouvelle minuscule wisigothique au début du XII siècle, sauf quelques exceptions, jusqu'au xnII $^{e}$ siècle, dans les livres liturgiques $^{42}$. Dans le tableau de lettres fourni par A. Duran Gudiol pour les inscriptions de la province de Huesca, cette forme du $\mathrm{T}$ ne dépasse pas 107243. Dans les inscriptions des Asturies et de León, je trouve le $T$ wisigothique entre 842 et 1108 . D'après Alphonse X le Sage, un concile, tenu à León en 1091, aurait ordonné de remplacer les lettres wisigothiques par des lettres françaises, gallicis litteris, mais nous n'avons pas d'autre témoignage d'un tel concile ${ }^{44}$. Même si on ne retient pas ce concile ou si l'on admet, ce qui semble logique, qu'une telle décision ne serait passée dans la pratique que progressivement, on doit aussi penser à une répercussion sur l'écriture wisigothique de l'abandon de la liturgie mozarabe, sous l'action de Grégoire VII et de son légat le cardinal Richard, abbé de Saint-Victor de Marseille - abandon confirmé par le roi Alphonse pour tout son royaume au concile de Burgos de $1080^{45}$-, et à l'influence française que véhiculèrent alors les chemins de Saint-Jacques et l'implantation des Clunisiens en Espagne46.

Les autres caractères épigraphiques renvoient tous à la fin du $\mathrm{xI}^{\mathrm{e}}$ ou au début du xire siècle ${ }^{47}$ : tous les $C$ sont carrés (C), les $O$ sont en navette $(\diamond)$, il n'y a aucune onciale. La ponctuation régulière par

41. Zacarias Garcia Villada, Paleografía española, Madrid, 1923, p. 243-244; en Navarre et en Aragon de la fin du $x^{e}$ aux premières années de la seconde moitiê du $x^{e}{ }^{e}$ siècle $^{2}$ (p. 251).

42. B. Bischoff, Paléographie de l'Antiquité romaine et du Moyen Âge occidental, Paris, 1985, p. 108 et 113 ; Anscari M. Mundo, * La datación de los códices liturgicos visigóticos toledanos ", Hispania sacra, XVIII, $\mathrm{n}^{\circ} 35,1965$, p. 21, cite un livre de Sahagan de 1110 et un livre d'Oviedo (1101-1129) comme les derniers grands exemples toujours mentionnes d'écriture wisigothique, et signale quelques emplois plus tardifs.

43. Antonio Duran Gudiol, Las inscripciones medievales de la provincia de Huesca, Saragosse, 1967, p. 7 (Estudios de la Edad media de la Corona de Aragon, 8).

44. Villada, op. cit., p. 88 ; Maurice Prou, Manuel de paléographie latine et francaise du VI $I^{e}$ au XVII siècle, $2^{e}$ ed., Paris, 1892, p. 39-41; L'art de vérifier les dates..., $3^{e}$ éd. I, Paris, 1783, p. 743.

45. Sacrosancta concilia, éd. Labbé et Cossart, Paris, X, 1671, c. 1815 et 229-231.

46. Marcelin Defourneaux, Les Frangais en Espagne aux XI' et XII' siècles, Paris, 1949, p. 17-124; Georges Gaillard, "La pénétration clunisienne en Espagne pendant la première moitié du XIe siècle *, et « Cluny et l'Espagne dans l'art roman du XIe siècle \#, dans Études d'art roman, Paris, 1972, p. $84-98$ (publications de la Sorbonne, série * Études *, t. 3). On peut encore ajouter que la façon de gagner de la place en raccourcissant une des parties de la lettre, sur la patène la première branche du $A$ de ULLA et de AMEN, du $V$ de VETUSTAS, du $M$ de AMEN, est propre à l'écriture des inscriptions de la péninsule. On trouve ce procédé dès 931 à Malaga en Andalousie et il est fréquent dans le Nord-Ouest de la péninsule, Asturies, Galice, jusqu'au xrre siècle, avec encore des exemples, plus mares, dans les Asturies jusqu'au début du XIVe siècle.

47. Paul Deschamps, *Étude sur la palégraphie des inscriptions lapidaires de la fin de l'époque mérovingienne aux dernières années du XII siècle $*$, Bulletin monumental, 88, 1929 , p. $5-81,35$ pl. h.t. 
trois points verticaux est davantage du $\mathrm{XII}^{\mathrm{e}}$ que $\mathrm{du} \mathrm{XI}^{\mathrm{e}}$ siècle, mais on la trouve déjà dans la croix de Peñalba au $x^{e}$ siècle. On arrive à la même datation lorsqu'on compare l'écriture des inscriptions de la patène et du calice de l'abbé Pélage avec les légendes en majuscules qui accompagnent les scènes peintes dans les manuscrits de la péninsule Ibérique à cette époque, et au premier lieu ceux de Compostelle ${ }^{48}$. C'est donc vers la fin du XI ${ }^{e}$ et le premier tiers du XII ${ }^{e}$ siècle qu'oriente l'étude paléographique. Le $\mathbf{T}$ wisigothique semblant avoir perdu son tracé habituel pour devenir presque décoratif, c'est plutôt à la fin de son emploi qu'il faut penser, donc au XII ${ }^{e}$ siècle.

Les historiens de l'art ont pu hésiter sur l'origine de ce calice et de cette patène, et, à partir de rapprochements avec un calice islandais conservé au Victoria and Albert Museum à Londres et le calice mosan de frère Bertin de 1222, conservé au musée des Cloîtres à New York, émettre, " prudemment ", l'hypothèse d'une possible origine mosane, ce que traduit la présentation de ces deux objets liturgiques au musée du Louvre ${ }^{49}$. Je crois qu'il faut vraiment retenir une origine espagnole - région d'Astorga-León -, du fait du nom de Pélage, de la formule d'offrande à saint Jacques, de l'écriture. D'ailleurs ce calice et cette patène, avant d'être acquis par Charles Stein, se trouvaient en possession du cardinal archevêque de Tọlède don Juan Ignacio Moreno, dont on peut rappeler qu'il fut d'abord évêque d'Oviedo puis de Valladolid.

Pour la datation Émile Molinier proposait " sans hésitation " la première moitié du XIII ${ }^{e}$ siècle dans son étude de 1888 , mais en 1901 soulignait l'influence française dans ce calice et cette patène et indiquait que forme et décoration conduisaient à des rapprochements avec des monuments fabriqués en France pendant la seconde moitié du

48. On notera en particulier qu'on retrouvera les mêmes types de caractères, à peine plus évolués, dans la première partie du cartulaire Tumbo A du trésor de la cathédrale de Compostelle, qui date de 1129-1133, alors que dans la partie suivante commencée à partir de la seconde moitié du XII siècle, l'écriture est radicalement nouvelle (Manuel C. Diaz y Diaz, Fernando Lopez Alsina, Serafin Moralejo Alvarez, Los tumbos de Compostela, Madrid, 1985). Cette comparaison m'a été suggérée par mon confrère et ami Jean Vezin, que je remercie vivement. Le Libro de los Testamentos de la Cathédrale d'Oviedo, composé pour l'évêque Pélage en 1126-1129 est d'une écriture plus avancée, avec nombre de belles onciales (Ars Hispaniae, XVIII, Miniatura, par Jesus Dominguez Bordona, Madrid, 1958, par exemple fig. 40). Si on consulte les Manuscrits enluminés de la péninsule Ibérique, par François Avril, Jean-Pierre Aniel, Mireille Mentré, Alix Saulnier, Yolanda Zaluska, Paris, 1982 (Bibliothèque nationale, Département des manuscrits, Centre de recherche sur les manuscrits enluminés), les rapprochements d'écriture se font avec les manuscrits du $\mathrm{XI}^{e}$ siècle.

49. J'ai soumis la question de datation à $M$. Piotr Skubiszewski, auteur de la remarquable étude citée ci-dessus, qui concerne 81 calices et patènes romans : la datation proposée à partir des inscriptions ne lui a semblé en rien contredite par la forme et le décor du calice et de la patène. 
XII siècles0. Dom Roulin, étudiant "le calice ministériel de Silos ", daté de 1041-1073, mentionne le calice de Pélage et le date du $\mathrm{XII}^{e}$-début XIII sièclesi. Marquet de Vasselot dans son catalogue donne, en 1914, la datation de fin XII $\mathrm{XI}^{\mathrm{e}}$ ébut $\mathrm{XIII}^{e}$ siècle52. Angela Franco Mata identifie Pélage avec l'évêque d'Astorga de ce nom (1097-1121) bien que le style du calice soit postérieur ${ }^{53}$. Je crois qu'il faut effectivement retenir une datation de la fin du $\mathrm{XI}^{e}$ ou de la première moitié du XII siècle, avec une préférence pour ce premier tiers ou mieux deuxième quart du XII siècle, où les derniers caractères wisigothiques vont disparaître et où va s'affirmer l'influence française que traduit probablement la forme du distique élégiaque léonin riche.

L'abbé Pélage serait-il l'évêque d'Astorga de 1097-1121 avant son accession à l'épiscopat ? Bien des évêques de ce temps ont été pris dans les monastères. Astorga a été une étape importante sur la route de Compostelle au point qu'elle compta vingt-cinq établissements hospitaliers. Pélage joua un rôle de premier plan dans le mouvement de retour à l'Église des biens usurpés par les laïcs. Il obtint ainsi en 1120 de la terrible reine Urraca, fille d'Alphonse VI, roi de Castille et de León, la restitution des différents monastères et biens qui avaient été enlevés à ses prédécesseurs, et en retour il lui fit don de "vases d'or et d'argent estimés à 2083 sous "54; il fit aussi des donations importantes à sa cathédrales5. On ne peut pour autant affirmer l'identité de l'abbé donateur du calice avec l'êvêque Pélage. León est un autre lieu privilégié de ce dossier. La ville est sur la route de Compostelle, elle est capitale de royaume. Le trésor de l'église San Isidoro conserve un calice, donné en 1063 par Urraca, fille de Ferdinand Ier, roi de León et de Castille $(+1065)$, et qui porte l'inscription : + IN NOMINe Domini URRACA FREdiNandi ${ }^{56}$. Un évêque Pélage siège à León de 1065 à 1085 . Quant à Santiago de Peñalba, on y connaît seulement, pour cette période, un abbé français Étienne, mort en 1132, et dont l'épitaphe en distiques élégiaques léonins riches comporte une série d'expressions qui se retrouvent dans des épitaphes

50. E. Molinier, * Le calice de l'abbé Pélage... *, p. 311 ; Histoire générale des arts appliqués à l'industrie..., IV, p. 195.

51. Dom E. Roulin, * Le calice ministériel de Silos ", dans Revue de l'art chrétien, 4" s., IX, 1898 , p. 360 .

52. J.-J. Marquet de Vasselot, Musée du Louvre. Orfèvrerie..., p. 7, n 24.

53. * E1 Diario de León *, 19 février 1989 (dossier musée du Louvre).

54. España sagrada..., XVI, ed. H. Florez, p. 194-197 et 477-479: * vasa argentea et aurea appreciata ad duo millia et octuaginta et tres solidorum ". La mère d'Urraca, Constance, était fille du duc Robert de Bourgogne, ct nièce d'Hugucs, abbé dc Cluny.

55. Ibid., p. 195-196.

56. E. Molinier, Histoire générale des arts..., IV, p. 102 (avec une mauvaise identification d'Urraca); Antonio Vinayo Gonzalez, L'ancien royaume de León roman, p. 105-106, pl. coul. après pl. 66. 
françaises des $\mathrm{XI}^{\mathrm{e}}$-XIII ${ }^{\mathrm{e}}$ siècles ${ }^{57}$. Cette épitaphe, c'est "Pelagius Fernandez " qui la commanda, "Petrus » qui l'écrivit. On pourrait aussi penser à ce Pélage s'il fut abbé après la mort d'Étienne, et le Petrus peut aussi bien être un Pedro qu'un Pierre. On reste évidemment là dans le domaine des hypothèses, mais c'est sans doute dans ce contexte Peñalba-Astorga-León de la première moitié du xire siècle, peut-être plus précisément de ces années 20 ou 30 où disparaît l'écriture wisigothique et apparaît le vers léonin riche dans les inscriptions, sous l'influence française dans les deux cas, que se placent le calice et la patène du musée du Louvre, avec l'affirmation - maladroite - d'une totale fídélité à une doctrine orthodoxe sur l'Eucharistie. Elle s'inscrit dans une riche série d'inscriptions relatives à ce sacrement ${ }^{58}$.

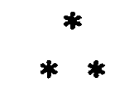

MM. Robert-Henri Bautier, Michel Mollat DU Jourdn, Francis SALET et Jacques FonTAINE interviennent après cette communication.

M. Jacques FONTAINE présente les observations suivantes :

Lecture, situation, datation sont très convaincantes; elles illustrent le savoir et la méthode de l'orateur - fondateur et animateur du remarquable Corpus des inscriptions médiévales de la France, dont le tome 16 vient de paraître en 1992. Peñalba est un haut lieu de rencontre entre les civilisations hispaniques, qu'illustre bien la pièce étudiêe : Thébaïde espagnole peuplée de moines depuis le vire siècle wisigothique (đans la fameuse "vallée du silence " située au-dessus du village), la vallée de Peñalba voit construire au $x^{e}$ siècle, par des moines venus d'Andalousie, la célèbre église préromane où l'on observe l'apport raffiné de techniques et de formes mozarabes; enfin cette église, à travers son titre de Santiago, témoigne du rayonnement européen du camino francés des pèlerins de Compostelle, qui passe au nord de Peñalba.

Deux traits appuient ces continuités et confirment ainsi la situation proposée pour les deux pièces : à Peñalba, à la fin du xie siècle. D'abord les erreurs de graphie : ne seraient-ce pas, en fait, des mélectures de lettres (confusion aisée de $r, s, n$ ) ou d'abréviations, sur un

57. Ainsi Moribus eximius, sub marmore, vir Domini, vivere dum licuit.

58. R. Favreau, * L'épigraphie comme source pour la liturgie *, dans Vom Quellenwert der Inschriften. Vorträge und Berichte der Fachtagung, Esslingen 1990, éd. Renate NeumullersKlauser, Heidelberg, 1992, p. 98-106 (Supplemente zu den Sitzungsberichten der Heidelberg Akademie der Wissenschaften, Philosophisch-historische Klasse). 
texte modèle rédigé en minuscule hispanique (ou * wisigothique $*$ ) ? Quant à l'Agneau mystique (cf. Apocalypse 14) figuré sur le fond de la patène, tourné de profil vers la gauche et tenant de sa patte droite repliée une croix wisigothique hampée, c'est un schème que l'on trouve dans la figuration d'Apocalypse 14 sur les miniatures " mozarabes " ( $\mathrm{x}^{\mathrm{e}} \mathrm{s}$. et suiv.) du commentaire hispanique de Béatus sur l'Apocalypse. Cet * Agneau mystique * figure aussi sur le couvercle d'une pièce d'orfevrerie du $x^{e}$ siècle : le coffret à reliques orné de métal repoussé, dont Alphonse III et la reine Chimène firent présent à la cathédrale d'Astorga - où l'on peut encore le voir. Ce n'est pas loin de Peñalba, dont les abbés ont entretenu des rapports suivis avec les évêques d'Astorga, comme il a été dit dans cette notable communication.

\section{LIVRES OFFERTS}

M. Jean Leclant a la parole pour deux hommages de la part de $\mathbf{M}$. Jean Pouilloux :

* L'amitié de notre Secrétaire perpétuel et de notre Président me permet de déposer in absentia sur le bureau de l'Académie, de la part de la Société des Études Chypriotes, le beau volume, qu'avec la collaboration de la fondation A. G. Levantis, elles viennent de consacrer en hommage à notre confrère de Chypre, Vassos Karageorghis : c'est un livre magnifique, de 374 pages in- $4^{\circ}$, accompagnées de 86 planches hors-texte, et auquel non moins de 50 historiens ou archéologues, issus de Chypre ou venus du monde entier, ont tenu à apporter une contribution, voulant ainsi témoigner leur gratitude et leur admiration à celui qui fut pendant trente ans l'âme de la recherche archéologique en son pays.

Fouilleur d'élite, que Sir Mortimore Wheeler se faisait gloire d'avoir formé, il fut pendant ces trente ans un directeur exigeant pour lui et pour les autres, constamment obsédé par la nécessité de faire participer la communauté scientifique aux découvertes exceptionnelles qu'il avait faites à Kition, à Salamine, à Palaipaphos, à Mâ, partout ailleurs dans cette île si riche d'un passé complexe, à la rencontre des mondes occidentaux et orientaux. Innombrables ont été à travers le monde ses exposés en grec, en français, en anglais, et la bibliographie placée en tête du volume ne compte pas moins de 353 mentions, livres ou articles portant sur toutes les périodes, de la préhistoire à la fin de l'Antiquité. Vassos Karageorghis a su non seulement réunir autour 